\title{
Diagnóstico por Imagem do Antepé de Pacientes com Artrite Reumatóide Inicial
}

\section{Imasing Diagnosis of Forefoot in Early Rheumatoid Arthritis Patients}

\author{
Armando Kihara ${ }^{(1)}$, Monique S. Konai( ${ }^{(2)}$ Jader Silva $^{(1)}$, Flávia A. Sakamoto ${ }^{(1)}$, Rozana Mesquita Ciconelli(2), \\ Jamil Natour ${ }^{(2)}$, Artur da Rocha Corrêa Fernandes ${ }^{(1)}$
}

A artrite reumatóide (AR) é uma doença sistêmica crônica que leva à destruição articular progressiva, com limitação funcional importante ${ }^{(1)}$.

O principal local de acometimento dessa doença é a sinóvia, que reveste a articulação. A sinovite resulta de um aumento na permeabilidade capilar com formação do pannus, sendo que a proliferação sinovial é um evento precoce no curso da doença que pode ser visto antes da destruição cartilaginosa ou óssea ${ }^{(2)}$.

$\mathrm{Na}$ artrite reumatóide inicial, o paciente geralmente apresenta edema ou dor no antepé em $80 \%$ a $90 \%$ dos $\operatorname{casos}^{(3,4)}$.

As articulações metatarsofalângicas (MTF) são freqüentemente as primeiras articulações a serem acometidas na AR típica ( $10 \%$ a $20 \%$ dos pacientes). As anormalidades radiológicas no antepé geralmente são as manifestações iniciais da doença, antecedendo as alterações na mão e no punho. Os achados mais precoces aparecem nas metatarsofalângicas, especialmente na quinta, e têm um predomínio pelas faces mediais, com exceção do $\mathrm{V}$ metatarsiano, no qual erosões no aspecto lateral são achados precoces da doença ${ }^{(4-6)}$.

Em um estudo de 1998, Belt et al. ${ }^{(7)}$ avaliaram prospectivamente 83 pacientes com diagnóstico precoce de $\mathrm{AR}(<6$ meses), e o número de erosões na $\mathrm{V}$ metatarsofalângica foi o dobro das outras articulações no início do estudo, além de demonstrar maior dano ao final do estudo ${ }^{(7)}$.

A radiografia simples, apesar de ser o exame de referência para a avaliação do dano articular (erosões ósseas, redução do espaço articular, osteopenia periarticular, cistos, subluxações ou anquiloses) e da sua progressão, pode não fornecer informações diagnósticas suficientes num estágio inicial, já que é necessário ocorrer perdas substanciais do osso para serem radiograficamente detectáveis ${ }^{(8-11)}$.
Em virtude das limitações da radiografia simples, outras modalidades de diagnóstico podem ser usadas para demonstrar alterações mais precoces ${ }^{(12)}$.

A ultra-sonografia (USG) apresenta boa sensibilidade para o diagnóstico de lesões de partes moles, avaliação de tendões, bem como de derrame articular, espessamento sinovial e erosões ósseas ${ }^{(13-17)}$.

Wakefield et $a l^{(18)}$ avaliaram a sensibilidade do ultra-som na caracterização de erosões nas MCF, quando comparado com a radiografia convencional, tendo a ressonância magnética (RM) como padrão-ouro, sendo caracterizadas 6,5 vezes mais erosões com a USG na doença inicial.

Por meio do ultra-som também é possível visualizar a vascularização do pannus e da sinóvia com o uso do Doppler ${ }^{(19)}$. Estudos recentes sugerem que o Power Doppler seja um método mais sensível do que o Doppler convencional na avaliação da atividade da doença, devido a sua maior sensibilidade na detecção de fluxos mais lentos ${ }^{(20,21)}$.

A RM permite uma avaliação detalhada do envolvimento de todas as estruturas acometidas na doença (membrana sinovial, coleções líquidas intra e extra-articulares, cartilagem, ligamentos, tendões e bainhas $)^{(17,22,23)}$. Fornece excelente resolução dos tecidos, e a possibilidade de cortes multiplanares permite diagnóstico de lesões mais precocemente do que nos outros métodos de imagem ${ }^{(24)}$.

Como resultado de diversas tecnologias em relação aos aparelhos de RM, existem algumas diferenças nas definições para os achados mais comuns da AR, como, por exemplo, erosões, edema ósseo, sinovite e tenossinovite, o que torna difícil a comparação. Entretanto, consensos internacionais de medidas de resposta de ensaios clínicos em reumatologia (Outcome Measurement in Rheumatology Clinicals Trials - OMERACT) forneceram recomendações de definições padronizadas ${ }^{(25,26)}$.

\footnotetext{
1. Departamento de Diagnóstico por Imagem da Universidade Federal de São Paulo (Unifesp).

2. Disciplina de Reumatologia da Unifesp.

Endereço para correspondência: Artur da Rocha Corrêa Fernandes, DDI/Unifesp, Rua Botucatu, 740, CEP 04023-900, São Paulo, SP.
} 
Devido ao impacto na qualidade de vida e à freqüência de acometimento do antepé na AR, o seu estudo por meio dos métodos de imagem para detectar alterações nas fases iniciais pode contribuir na decisão da melhor abordagem para esses pacientes.

Descreveremos a seguir os achados mais comuns nas articulações MTF de pacientes portadores de AR em fase inicial aos diferentes métodos de imagem: radiografia simples, USG e RM.

Na Figura 1, encontram-se as imagens do antepé de um paciente portador de AR há aproximadamente dois anos. Observa-se que, pela radiografia simples e RM, não foram detectadas alterações. Entretanto, o exame de ultra-som detectou erosões na V MTF direita.

Por outro lado, o paciente da Figura 2 apresentou alterações aos três métodos de imagem. Na radiografia simples, podem-se observar erosões na I e V MTF. Essas erosões se repetem ao exame de ultra-som, que demonstra também
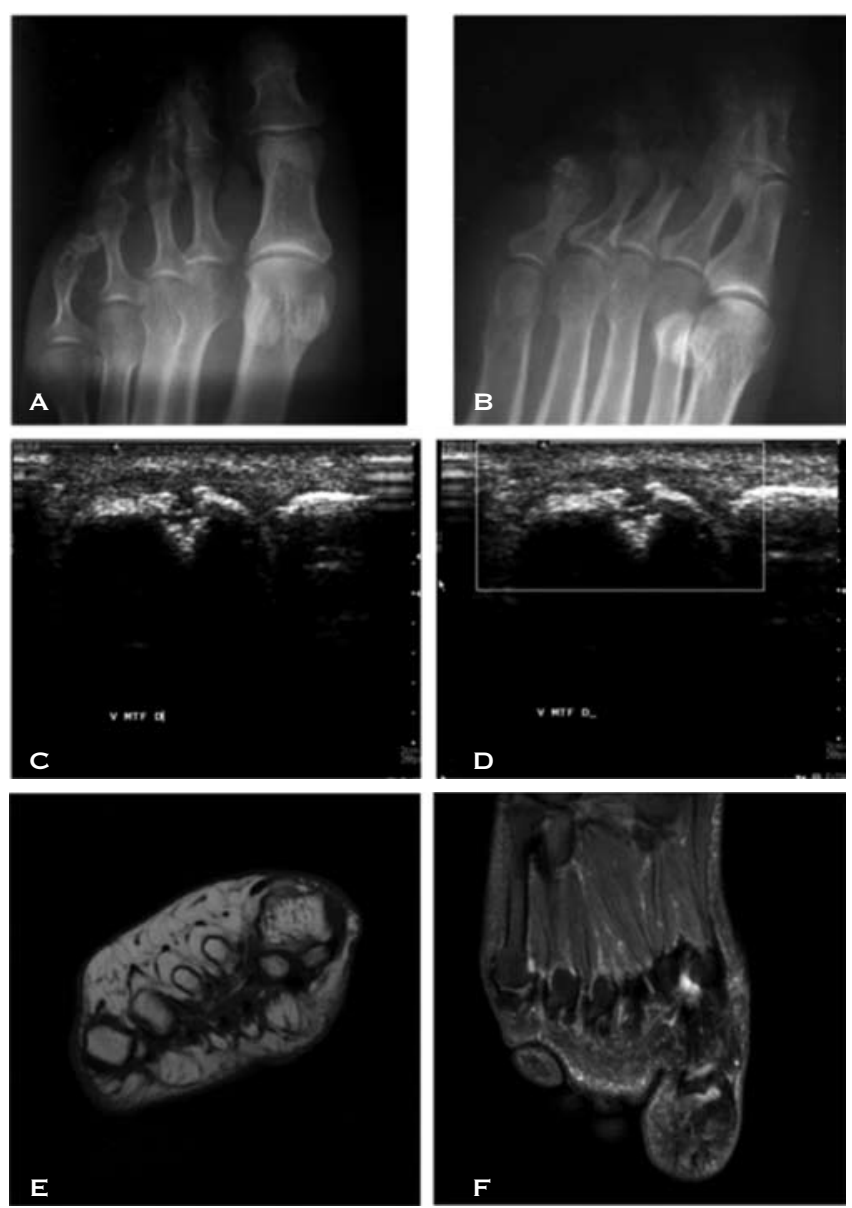

Figura 1 - Paciente de 43 anos. Na radiografia simples, não foram observadas alterações. No exame USG, foi caracterizada erosão na V MTF direita, sem sinais de sinovite. Na RM, não foram caracterizadas alterações. a presença de sinovite. Com o uso do Doppler, observou-se um aumento da vascularização local. Nas imagens fornecidas pela RM, além das erosões, nota-se a presença de sinovite evidenciada pela fase de contraste, conforme ilustrado nas imagens I e J da Figura 2.
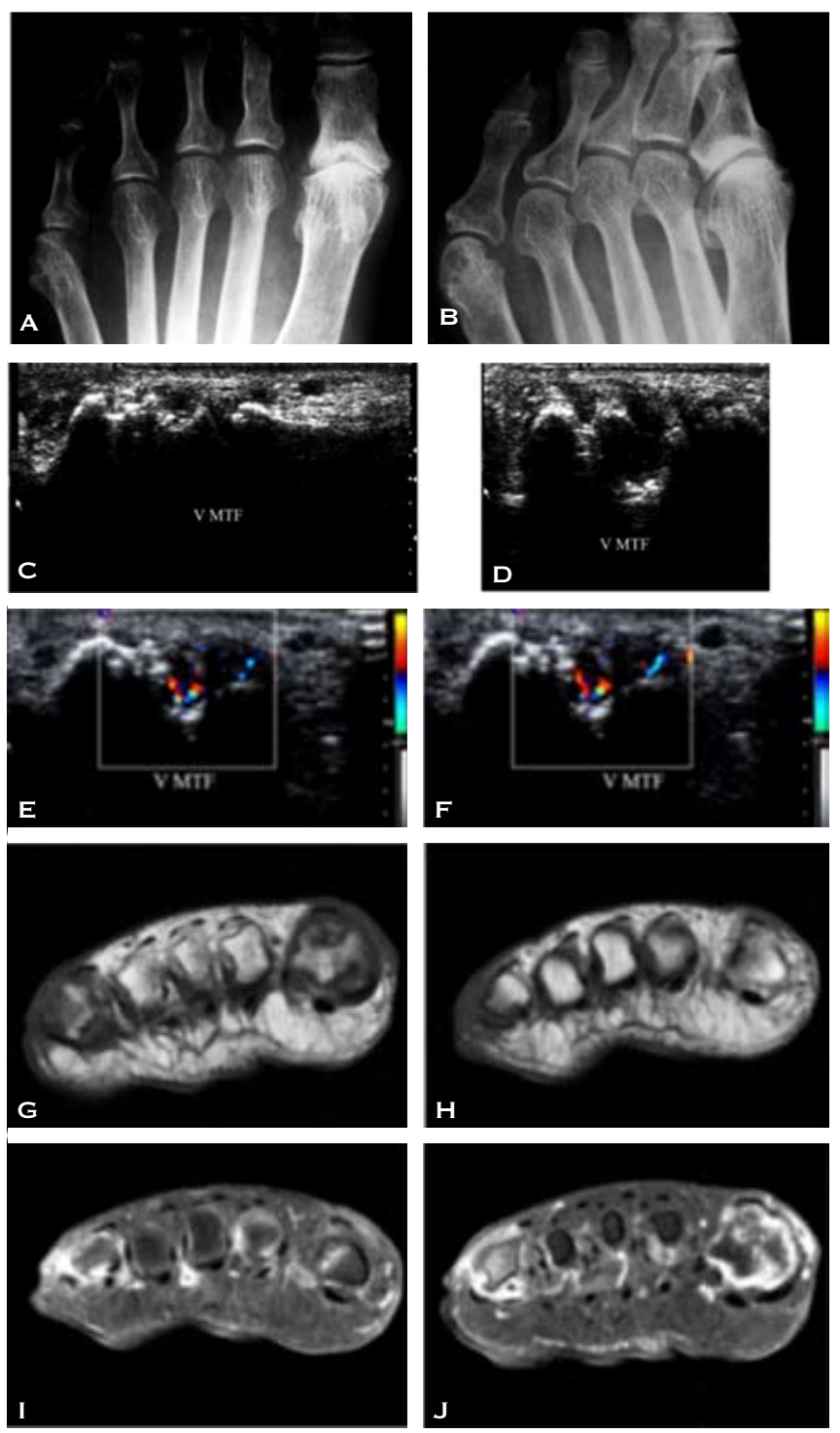

Figura 2 - Paciente de 57 anos, com erosões na I e V MTF, caracterizadas aos três métodos de imagem. Notam-se, ainda, sinais de sinovite caracterizados à USG e à RM.

Considerando a RM como padrão-ouro para a detecção das alterações nas MTF, observa-se que a USG apresenta resultados semelhantes, principalmente na avaliação da I e V MTF, tendo menor eficácia na caracterização de sinovite e principalmente erosões, nas demais articulações do antepé, provavelmente devido à dificuldade de avaliação de todas as superfícies articulares. 
Erosões marginais, osteopenia periarticular e edema de partes moles geralmente identificados à radiografia convencional são alterações habitualmente encontradas cerca de um a dois anos após o início dos sintomas articulares ${ }^{(11,23)}$. As erosões iniciam-se nas chamadas "áreas nuas" da articulação, isso é, superfícies ósseas não cobertas por cartilagem $\operatorname{articular}^{(4)}$. Nas articulações metatarsofalângicas, $\mathrm{o}$ aspecto medial é mais freqüentemente acometido, com exceção do V metatarsiano, em que o aspecto lateral é mais acometido ${ }^{(27)}$.

A avaliação do dano estrutural é fundamental porque alguns estudos demonstraram que a gravidade das alterações está associada a uma perda funcional irreversível e também porque o dano articular reflete o histórico do paciente em relação ao tratamento e à evolução da doença ${ }^{(28)}$.

A terapia precoce com drogas modificadoras da história natural da doença é fundamental para melhorar a evolução radiológica e funcional dos pacientes e tem como finalidade a supressão completa do processo inflamatório articular, reduzindo o dano estrutural e funcional. Para isso, são necessários um diagnóstico precoce e um bom método para acompanhar a progressão da doença $a^{(9,10,29,30)}$.

Apesar de a decisão da terapêutica não ser baseada na presença ou não de erosões, o diagnóstico precoce de alterações articulares pode auxiliar na escolha do tratamento.

A USG aparece como um bom método na avaliação de pacientes com AR inicial, pois, ao contrário da radiografia simples, permite a avaliação de alterações inflamatórias, especialmente sinovite e tenossinovite. Pode, ainda, ser associada ao uso de Doppler, com o qual é possível detectar sinais de neovascularização do pannus, cuja importância foi avaliada em alguns estudos que demonstraram que a redução da inflamação estava associada à menor destruição óssea e cartilaginosa ${ }^{(11,31,32)}$.

Quando comparada com a ressonância, a USG apresenta sensibilidade semelhante para a avaliação de articulações de fácil acesso. Como a USG não pode atravessar o osso, algumas superfícies articulares são inacessíveis.

No estudo de Wakefield et al.(18), a USG mostrou-se mais eficiente do que a radiografia simples na caracterização de erosões, principalmente nas fases iniciais da doença. A superioridade do ultra-som foi explicada pela sua capacidade multiplanar

\section{REFERÊNCIAS}

1. Gordon DA, Hastings DE: Clinical features of rheumatoid arthritis. In: Hochberg MC, Silman AJ, Smolen JS, Weinblatt ME, Weisman MH. Rheumatology 3.ed., 1: 765-780, 2003.

2. Firestein GS: Rheumatoid synovitis and pannus. In: Hochberg MC, Silman AJ, Smolen JS, Weinblatt ME, Weisman MH. Rheumatology 3.ed., 1: 855-84, 2003. e de detectar erosões menores. Quando comparados os resultados entre o ultra-som e a ressonância, dez erosões caracterizadas na RM correspondiam exatamente às alterações encontradas no ultra-som. Três erosões foram caracterizadas apenas na USG, esse fato se deve a uma resolução espacial superior da USG e à possibilidade de efeitos de volume parcial na RM.

Em outros estudos realizados com o ultra-som, confirmou-se a freqüência do acometimento do aspecto lateral da V MTF nos pacientes com $\operatorname{AR}^{(33-35)}$.

As vantagens da USG incluem fácil acesso, baixo custo, exame não-invasivo, ausência de radiação ionizante, capacidade de caracterizar alterações inflamatórias e destrutivas. As desvantagens são a necessidade de examinador experiente, difícil documentação e reprodutibilidade.

A RM aparece como método muito sensível na avaliação das alterações precoces da $\mathrm{AR}$, além de caracterizar erosões mais precocemente do que a radiografia convencional ${ }^{(12,36)}$.

A presença de sinais de edema da medular óssea é freqüente, essa alteração reversível parece estar associada com a formação de erosões, portanto, pode ser uma alteração ainda mais precoce na evolução da AR. Outra vantagem da ressonância é a capacidade de avaliar a cartilagem, o que é mais específico do que a avaliação indireta da redução do espaço $\operatorname{articular}^{(22)}$.

Em um estudo de punhos de 42 pacientes com AR precoce seguidos por dois anos, McQueen et al. ${ }^{(37)}$ demonstraram que as erosões na $\mathrm{RM}$ precederam as da radiografia em um a dois anos.

A sinovite é mais bem caracterizada com o uso do contraste paramagnético (gadolíneo) na RM, quando se comparam as seqüências pré e pós-contraste ${ }^{(38)}$. O realce sinovial pelo gadolíneo foi correlacionado com achados macro e microscópicos de inflamação em joelhos em pacientes com $\operatorname{AR}^{(38,39)}$.

Conaghan et al. ${ }^{(40)}$ conseguiram demonstrar que a progressão das erosões foi proporcional ao grau de sinovite, e não foram observadas erosões em articulações sem sinovite ${ }^{(40)}$.

Desvantagens da RM incluem altos custos e acesso restrito, mais tempo de exame, desconforto, restrição da avaliação a algumas articulações por exame e menos dados a respeito da padronização e valor prognóstico em relação à radiografia convencional.

\section{Declaramos a inexistência de conflitos de interesse.}

3. Solomon G: Inflammatory arthritis. In: Jahss MH (ed): Disorders of the foot and ankle: Medical and surgical management. 2.ed. Philadelphia: W.B. Saunders, 1991; pp. 1681-702.

4. Resnick D, Niwayama G: Rheumatoid Arthritis. In: Resnick D (ed): Diagnosis of bone and joint disorders. 3.ed. Philadelphia: W.B. Saunders, 1995; pp. 966-70. 
5. Kerr LD: Arthritis of the forefoot: A review from a rheumatologic and medical perspective. Clinical Orthopaedics and Related Research 349: 20-27, 1998.

6. Harris Jr. ED: Rheumatoid arthritis: Pathophysiology and implications for therapy. N Engl J Med 322: 1277-89, 1990.

7. Belt EA, Kaarela K, Lehto MUK: Destruction and arthroplasties of the metatarsophalangeal joints in seropositive rheumatoid arthritis. Scand J Rheumatol 27: 194-6, 1998.

8. Boers M, Verhoeven AC, Markusse HM, et al: Randomised comparison of combined step-down prednisolone, methotrexate and sulphasalazine with sulphalazine alone in early rheumatoid arthritis. Lancet 350: 309-18, 1997.

9. Möttönen T, Hannoven P, Leirisalo-Repo M, et al: Comparison of combination therapy with single-drug therapy in early rheumatoid arthritis: a randomised trial. Lancet 353: 1568-73, 1999.

10. Emery P. The optimal management of early rheumatoid disease: the key to preventing disability. Br J Rheumatol 33: 765-8, 1994

11. Lopez-Ben L, Bernreuter WK, Moreland LW, Alarcon GS. Ultrasound detection of bone erosions in rheumatoid arthritis: a comparison to routine radiographs of the hands and feet. Skeletal Radiol 33: 80-4, 2004

12. Backhaus M, Kamradt T, Sandrock D, Loreck D, Fritz J, Wolf $\mathrm{KJ}$, et al: Arthritis of the finger joints: a comprehensive approach comparing conventional radiography, scintigraphy, ultrasound, and contrast-enhanced magnetic resonance imaging. Arthritis Rheum 42: 1232-45, 1999.

13. Hau M, Schultz H, Tnoy H-P, Keberle M, Jahns R, Haerten $\mathrm{R}$, Jennet $\mathrm{M}$ : Evaluation of pannus and vascularization of the metacarpophalangeal and proximal interphalangeal joints in rheumatoid arthritis by high-resolution ultrasound. Arthritis Rheum 42(11): 2303-8, 1999.

14. De Flaviis L, Scaglione P, Nessi R, Ventura R, Calori G: Ultrasonography of the hand in rheumatoid arthritis. Acta Radiol 29: 457-60, 1988.

15. Grassi W, Tittarelli E, Pirani O, Avaltroni D, Cervini C: Ultrasound examination of metacarpophalangeal joints in rheumatoid arthritis. Scand J Rheumatol 22: 243-7, 1993.

16. Lund PJ, Heikal A, Maricic MJ, Krupinski EA, Williams CS: Ultrasonographic imaging of the hand and wrist in rheumatoid arthritis. Skeletal Radiol 24: 591, 1995.

17. Fessel DP, Jamadar DA, Jacobson JA, Caoili EM, Dong Q, et al: Sonography of dorsal ankle and foot abnormalities. AJR 181: 1573-81, 2003.

18. Wakefield RJ, Gibbon WW, Conaghan PG, et al: The value of sonography in the detection of bone erosions in patients with rheumatoid arthritis. Arthritis Rheum 43: 2762-70, 2000.

19. Terslev L, Torp-Pedersen S, Qvistgaard E, Bliddal H: Spectral Doppler and resistive index. Acta Radiol 44: 645-52, 2003.

20. Wakefield RJ, Brown AK, O'Connor PJ, Emery P: Power Doppler Sonography: Improving disease activity assesment in inflammatory musculoskeletal disease. Arthritis Rheum 48(2): 285-88, 2003

21. Szkudlarek M, Court-Payen M, Strandberg C, Klarlund M, Klausen T, Ostergaard M: Power Doppler ultrasonography for assessment of synovitis in the metacarpophalangeal joints of patients with rheumatoid arthritis. Arthritis Rheum 44(9): 2018-23, 2001.

22. Ostergaard M, Szkudlarek M: Imaging in rheumatoid arthritis - why MRI and ultrasonography can no longer be ignored. Scand J Rheumatol 32: 63-73, 2003.
23. Larsen A, Dale K, Eek M: Radiographic evaluation of rheumatoid arthritis and related conditions by standard reference films. Acta Radiol 18: 481-91, 1977.

24. Rosenberg ZS, Beltran J, Bencardino JT: MR Imaging of the Ankle and Foot. Radiographics 20: s153-79, 2000.

25. Conagham P, Edmons J, Emery P, et al: Magnetic resonance imaging in rheumatoid arthritis: Summary of OMERACT activities, current status and plans. J Rheumatol 28: 1158-62, 2001.

26. Ostergaard M, Peterfy C, Conagham P, et al: Core set of MRI acquisitions, joint pathology definitions and scoring system (OMERACT 2002 RAMRIS). J Rheumatol 30: 1385-6, 2003.

27. Fleming A, Benn RT, Corbett M, Wood PHN: Early rheumatoid disease: II. Patterns of joint involvement. Ann Rheum Dis 35: 361-64, 1976.

28. Landewé $\mathrm{R}$, Heijde $\mathrm{D}$ : Is radiographic progression a realistic outcome measure in clinical trials with early inflammatory arthritis? Clin Exp Rheumatol 21(s31): s37-41, 2003.

29. Barthon JM, Martin RW, Fleischmann RM, Tesser JR, Schiff MH, et al: A comparison of etanercept and methotrexate in patients with early rheumatoid arthritis. N Engl J Med 343: 1586-93, 2000.

30. Treatment of poor-prognosis early rheumatoid arthritis - a randomized study of treatment with methotrexate, cyclosporin A, and intraarticular corticosteroids compared with sulfasalazine alone. Arthritis Rheum 48: 1809-19, 2000.

31. Peacock DJ, Banquerigo ML, Brahn E: Angiogenesis inhibition suppresses collagen arthritis. J Exp Med 175: 1135-38, 1992.

32. Arsenault AL, Lhotak S, Hunter WL, Banquerigo ML, Brahn E: Taxol involution og collagen-induced arthritis: ultrastructural correlation with the inhibition of synovitis and neovascularization. Clin Immunol Immunopathol 86: 280-89, 1998.

33. Alarcón GS, Lopez-Ben R, Moreland LW: High-resolution ultrasound for the study of target joints in rheumatoid arthritis. Arthritis Rheum 46: 1969-81, 2002.

34. Grassi W, Filipucci E, Farina A, Salaffi F, Cervini C: Ultrasonography in the evaluation of bone erosions. Ann Rheum Dis 60: 98-103, 2001.

35. Klocke R, Glew D, Cox N, Blake DR: Sonographic erosions of the rheumatoid little toe. Ann Rheum Dis 60: 896-7, 2001.

36. Corvetta A, Giovagnoni A, Baldelli $S$, et al: MR imaging of rheumatoid hand lesions: comparison with conventional radiology in 31 patients. Clin Exp Rheumatol 10: 217-22, 1992.

37. McQueen FM, Benton N, Crabbe J: What is the fate of erosions in early rheumatoid arthritis? Tracking individual erosions using $\mathrm{x}$-rays and magnetic resonance imaging over the first two years of disease. Ann Rheum Dis 60: 859-68, 2001.

38. Ostergaard $M$, Klarlund $M$ : Importance of timing of postcontrast MRI in rheumatoid arthritis: what happens during the first 60 minutes after IV gadolinium-DTPA? Ann Rheum Dis 60: 1050-54, 2001.

39. Tamai K, Yamato M, Yamaguchi T, Ohno W: Dynamic magnetic resonance imaging for evaluation of synovitis in patients with rheumatoid arthritis. Arthritis Rheum 37: 1151-57, 1994.

40. Conaghan PG, O'Connor P, McGonagle D: Elucidation of the relationship between synovitis and bone damage: a randomized MRI study of individual joints in patients with early rheumatoid arthritis. Arthritis Rheum 48: 64-71, 2003. 